\title{
EFFECTS OF TURBULENCE-RADIATION INTERACTIONS IN A NON-PREMIXED TURBULENT METHANE-AIR FLAME
}

\author{
L. D. Lemos, \\ F. R. Centeno, \\ and F. H. R. França \\ Universidade Federal do Rio Grande do Sul \\ Departamento de Engenharia Mecânica \\ Bairro Centro Histórico \\ CEP. 90050-170, Porto Alegre, RS, Brasil \\ larissa.lemos@ufrgs.br \\ Received: April 14, 2018 \\ Revised: May 11, 2018 \\ Accepted: May 31, 2018
}

\section{ABSTRACT}

This work studied a turbulent flame and analyzed the interaction between turbulence and radiation (TRI). The problem consists of a non-premixed turbulent methane flame surrounded by a low-velocity air coflow identified as Flame DLR-A. The steady laminar diffusion flamelet (SLDF) model is used to solve the chemical kinetics. To generate the flamelet library, turbulence-chemistry interaction is taken into account through previously assumed probability density functions (PDF) of mean scalars. Radiative heat flux is calculated with the discrete ordinates method, considering the Gray Gas model (GG). Turbulence is solved with k- $\varepsilon$ Standard model and TRI methodology is based on temperature self-correlation. The solution is obtained using ANSYS/Fluent code coupled with user-defined functions (UDFs). Results indicated that the temperature and chemical species predictions are little affected by TRI, while the radiative quantities (radiative heat flux on the domain wall) are importantly affect by TRI effects.

Keywords: turbulence-radiation interaction, gray gas model, flame dlr-a, sldf, cfd, user defined function

\section{NOMENCLATURE}

I radiation intensity, $\mathrm{W} / \mathrm{m}^{2}$

$\mathrm{k}$ turbulent kinetic energy, $\mathrm{m}^{2} / \mathrm{s}^{2}$

$\mathrm{T}$ temperature, $\mathrm{K}$

$\mathrm{T}^{\prime 2} \quad$ variance of temperature, $\mathrm{K}^{2}$

u axial velocity, $\mathrm{m} / \mathrm{s}$

$\mathrm{v}$ radial velocity, $\mathrm{m} / \mathrm{s}$

\section{Greek symbols}

$\varepsilon \quad$ dissipation rate of turbulent kinetic energy, $\mathrm{m}^{2} / \mathrm{s}^{3}$

$\kappa \quad$ absorption coefficient, $\mathrm{m}^{-1}$

$\rho$ density, $\mathrm{kg} / \mathrm{m}^{3}$

$\mu \quad$ viscosity, $\mathrm{Ns} / \mathrm{m}^{2}$

$\mu_{\mathrm{t}} \quad$ turbulent viscosity, $\mathrm{Ns} / \mathrm{m}^{2}$

\section{Subscripts}

$\mathrm{t}$ turbulence

b black body

\section{INTRODUCTION}

Turbulence-radiation interactions (TRI) have been revealing their importance for radiative heat transfer analysis. Studies have evidenced the inaccuracy of predictions of radiative heat flux and temperature when the TRI is neglected Centeno, et al., (2016) and Gupta, et al., (2013). Turbulent flames are present in several industrial applications. This is due to the participation of the turbulence together with the chemical reactions promoting the mixing of the reagents and the transport of heat. In addition, non-premixed flames are more commonly used because they allow significantly easier control than premixed flames. Since this is a complex problem involving highly nonlinear functions, the solution involves some methodologies to obtain accurate results.

The steady laminar diffusion flamelet (SLDF) was formalized by Peters (1984). The flamelets model treats a multidimensional flame as a group of structures of unidimensional laminar flames. Each flamelet is submitted to local conditions of flow, keeping its internal structure. In this way, the laminar structure of flamelets can be previously calculated from a database and then tabulated. The table contains the most important reactive scalars parametrized by a small number of control variables. The application of this methodology in several works has been shown to provide accurate results when compared with experimental data (Emami and Fard, 2012). In order to obtain the laminar flamelets, the chemical kinetic mechanism used is GRI-Mech 3.0 with 53 chemical species and 325 elementary reactions.

There are different turbulence models based on Reynolds-Average Navier-Stokes equations (RANS). Even with advanced methodologies as Large Eddy Simulation (LES), RANS approach is still being broadly employed. This is due its relative easiness of implementation and convergence. In this work the closure model is Standard $\mathrm{k}-\varepsilon$, which showed accurate results for flame DLR-A according recent work (Deon, 2016).

Simulations of turbulent reactive flows are not satisfactorily computed with the scalars mean fields, so it is necessary a model that consider the effect of their fluctuations. Probability Density Function (PDF) has been provided reliable results in combustion problems (Ziani, et al., 2013). The function probability density can be interpreted as the 
fraction of time which a portion of fluid in determined spatial coordinate stays in the neighbourhood of a state. The advantage of PDF models is the dependency of probability function with the real fluctuations. In this model the mean and the variance are the key parameters of the probability function. The turbulence effects are incorporated in laminar flamelets through PDFs.

The radiative heat transfer was calculated with the discrete ordinates method (DO) being used 60 directions, the spectral analysis was made by the Gray Gas model (GG). The GG model considers that the coefficient of absorption of the participant media does not dependent on the wave number and it is possible assume a constant value for the entire spectrum. The GG model was applied several times (Cassol et al., 2015) due the simplicity of the method. The TRI analysis is made through the radiative transfer equation (RTE). The RTE is applicable only to instantaneous quantities, while the turbulence model employed in the present study provides only mean temporal quantities. In this way it is necessary the decomposition of the variables in medium and fluctuating components that require modelling (Coelho, 2007). In this work the approximation used was proposed by Snegirev (2004). It considers the combined correlation between the absorption coefficient and the temperature and the temperature self-correlation. These two correlations of TRI interactions were considered the most important in reactive flows ( $\mathrm{Li}$ and Modest, 2002a; Li and Modest 2002b; Gupta, et al., 2013). In this way, the objective of this work is to evaluate the contribution of the TRI in DLR-A flame.

\section{THEORY}

The problem was solved using ANSYS Fluent, academic version 18 . The solution was built setting a series of parameters for Standard $k-\varepsilon$, builting the flamelets and creating a PDF Table (Ansys, 14.0 2011a.). This is a sequence of steps available on Fluent. The TRI study needs special implementation, so, the radiative heat transfer solution demanded User Defined Functions (UDF's), programmed according Ansys 14.0 (2011b). Several UDF's were programmed and coupled to Ansys Fluent, including codes for variance of temperature, TRI modeling, GG modeling, RTE solution, and the coupling between them and the main code originally available on Ansys Fluent.

\section{Mathematical Equations}

Considering the spectrally integrated RTE and averaging it in time results in:

$$
\frac{\mathrm{d} \overline{\mathrm{I}}}{\mathrm{dS}}=-\overline{\kappa \mathrm{I}}+\overline{\kappa \mathrm{I}_{\mathrm{b}}}
$$

The first term on the right side in Eq. (1) considers the cross-correlation between the absorption coefficient, $\kappa$, and the radiation intensity, I, which can be written by the following equation. The Ib is the intensity of radiation of a black body.

$$
\overline{\kappa \mathrm{I}}=\bar{\kappa} \overline{\mathrm{I}}+\overline{\kappa^{\prime} \mathrm{I}^{\prime}}
$$

The Optically Thin Fluctuation Approximation (OTFA) allows assume that $\overline{\kappa^{\prime} \mathrm{I}^{\prime}} \approx 0$, leading to:

$$
\frac{\mathrm{d} \overline{\mathrm{I}}}{\mathrm{dS}}=-\bar{\kappa} \overline{\mathrm{I}}+\overline{\kappa \mathrm{I}_{\mathrm{b}}}
$$

The second term on the right side is proportional to $\overline{\kappa \mathrm{T}^{4}}$, the approximation proposed by Snegirev is:

$$
\overline{\kappa \mathrm{T}^{4}}=\bar{\kappa} \cdot \overline{\mathrm{T}}^{4}\left(1+\mathrm{C}_{\mathrm{TRI} 1} 6 \frac{\overline{\mathrm{T}^{\prime 2}}}{\overline{\mathrm{T}}^{2}}+\left.\mathrm{C}_{\mathrm{TRI} 2} 4 \frac{\overline{\mathrm{T}^{\prime 2}}}{\bar{\kappa} \cdot \overline{\mathrm{T}}} \frac{\partial \kappa}{\partial \mathrm{T}}\right|_{\overline{\mathrm{T}}}\right)
$$

The constants $\mathrm{C}_{\mathrm{TR} I 1}$ and $\mathrm{C}_{\mathrm{TRI} 2}$ are respectively 2.5 and 1.0. Some steps have been skipped for brevity, being described in detail in Centeno (2014). The main advantage of this TRI methodology is that only one additional variable needs to be determined, the variance of temperature. To obtain this variable, the following transport equation must be solved:

$$
\begin{aligned}
& \frac{\partial}{\partial \mathrm{z}}\left(\rho \overline{\mathrm{u}} \overline{\mathrm{T}^{\prime 2}}\right)+\frac{1}{\mathrm{r}} \frac{\partial}{\partial \mathrm{r}}\left(\mathrm{r} \rho \overline{\mathrm{v}} \overline{\mathrm{T}^{\prime 2}}\right)= \\
& \frac{\partial}{\partial \mathrm{z}}\left(\left(\mu+\frac{\mu_{\mathrm{t}}}{\mathrm{Pr}_{\mathrm{t}}}\right) \frac{\partial \overline{\mathrm{T}^{\prime 2}}}{\partial \mathrm{z}}\right)+\frac{1}{\mathrm{r}} \frac{\partial}{\partial \mathrm{r}}\left(\mathrm{r}\left(\mu+\frac{\mu_{\mathrm{t}}}{\mathrm{Pr}_{\mathrm{t}}}\right) \frac{\partial \overline{\mathrm{T}^{\prime 2}}}{\partial \mathrm{r}}\right) \\
& +2 \frac{\mu_{\mathrm{t}}}{\operatorname{Pr}_{\mathrm{t}}}\left(\frac{\partial \overline{\mathrm{T}}}{\partial \mathrm{z}}+\frac{\partial \overline{\mathrm{T}}}{\partial \mathrm{r}}\right)^{2}-\mathrm{C}_{\mathrm{T}} \rho \overline{\mathrm{T}^{\prime 2}} \frac{\varepsilon}{\mathrm{k}}
\end{aligned}
$$

The transport equation was calculated by ANSYS Fluent through UDF's. According Ansys 14, $2011 \mathrm{~b}$ it is possible to calculate any transport equation for a scalar just enabling the equation and setting or programming terms of source and diffusion according the problem. Similarly, the implementation of RTE was built directly increasing the emission term. However, in this case, the GG model uses a constant absorption coefficient and this leads to zero the derivative term of Equation (4). Thereby, the emission term of RTE is affected by TRI according the following equation:

$$
\overline{\kappa \mathrm{T}^{4}}=\bar{\kappa} \cdot \overline{\mathrm{T}}^{4}\left(1+\mathrm{C}_{\mathrm{TRI} 1} 6 \frac{\overline{\mathrm{T}^{\prime 2}}}{\overline{\mathrm{T}}^{2}}\right)
$$

An UDF for implementation of the RTE, and for 
set the source term in Eq. (5), consists in small routines written on $\mathrm{C}$ programming language that can be coupled with Fluent in order to modify the models or change variables treatments.

The constant absorption coefficient for entire spectrum is absolutely an approximation. The next step of this study is the implementation of WeightedSum-of-Gray-Gases model (WSGG) model. The WSGG model considers that the entire spectrum can be divided into a few gray gases with constant absorption coefficient plus one transparent window. Being this the first implementation of TRI approach on Ansys Fluent, the GG model was chosen due the simplicity.

\section{DLR-A Flame}

A non-premixed turbulent flame of a mixture of methane $\left(\mathrm{CH}_{4}\right)$, hydrogen $\left(\mathrm{H}_{2}\right)$ and nitrogen $\left(\mathrm{N}_{2}\right)$ surrounded by a low-velocity coflow of air (TNF Workshop, 1998) has been proposed by the DLR Institute of Combustion Technology from Stuttgart (Bergmann, 1998). Posteriorly, two other laboratories conducted similar experiments, the Sandia National Laboratory from Livermore (Meier et al., 2000), and the Technische Universität Darmstadt (Schneider et al., 2003).

The flame consists of a fuel mixture of $22.1 \%$ $\mathrm{CH}_{4}, 33.2 \% \mathrm{H}_{2}$ and $44.7 \% \mathrm{~N}_{2}$ (volume), supplied by a vertical stainless steel tube with internal diameter, $\mathrm{D}=8 \mathrm{~mm}$ with a thinned rim at the exit, and a $350 \mathrm{~mm}$ long straight cross-section prior to exit. Its bulk velocity, $42.15 \mathrm{~m} / \mathrm{s}$, corresponds to a Reynolds number equal to 15200 based on internal diameter of the pipe. Its stoichiometric mixture fraction is $Z s t=0.167$ and its adiabatic flame temperature is $\mathrm{Tad}=2130 \mathrm{~K}$. The geometrical domain consists, longitudinally, $40 \mathrm{~cm}$ prior to fuel jet and air coflow exit and more $200 \mathrm{~cm}$ since it, and $40 \mathrm{~cm}$, radially, as shown in Figure 1.

The air stream is provided by a forced-draft vertical wind tunnel, and has a velocity of $0.3 \mathrm{~m} / \mathrm{s}$. In the case of DLR Institute of Combustion Technology and Technische Universität Darmstadt, dry air is discharged by a circular nozzle, with an internal diameter of $140 \mathrm{~mm}$. In the case of Sandia National Laboratory experiments, air stream has $0.8 \%$ water vapor by volume and its outlet nozzle is square and $300 \mathrm{~mm}$ wide, with the fuel pipe centered on it. This difference between the air coflow suppliers, however, may not be significant, since its velocity is very low, being the room air the responsible for providing most of the oxidant needed by the flame.

Fuel and oxidant streams and the environment are maintained at the temperature of $292 \mathrm{~K}$. Ambient pressure is reported to be $95.3 \mathrm{kPa}$ on DLR Institute of Combustion Technology, and $99.0 \mathrm{kPa}$ in the case of Sandia National Laboratory. In this work the original geometrical configuration and experimental conditions were adopted, as reported by DLR
Institute of Combustion Technology.

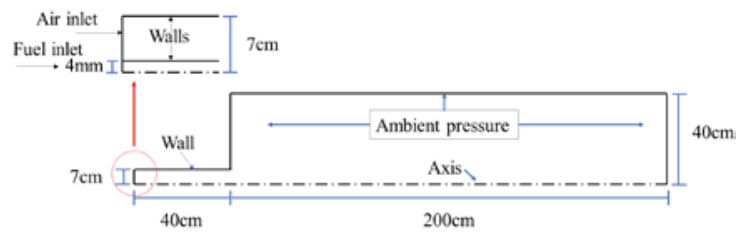

Figure 1. Axisymmetric representation of the geometrical domain and boundary conditions.

The Sandia National Laboratories produced experimental data for flame DLR-A (http://www.sandia.gov/TNF/DataArch/DLRflames.h tml, access in September 2017). There are measurements of temperature and mole fractions of $\mathrm{N}_{2}, \mathrm{O}_{2}, \mathrm{CO}_{2}, \mathrm{H}_{2} \mathrm{O}, \mathrm{H}_{2}, \mathrm{CO}, \mathrm{OH}$, and $\mathrm{NO}$ at axial positions ( $\mathrm{x} / \mathrm{d}=2.5$ up to $\mathrm{x} / \mathrm{d}=120)$ and radial positions ( $\mathrm{x} / \mathrm{d}=5,10,20,40,60,80)$, both mean and root mean square (rms) values. The work produced by Deon (2016) compared the numerical and experimental data and shows results that indicate independence with respect to the mesh refinement level. It is a two-dimensional mesh with rectangular non-uniform size elements, refinement concentrated at the region near the fuel jet. This is the same mesh used in the present work with 25,031 elements.

\section{Convergence criterion}

Simulations were performed using the commercial CFD code ANSYS Fluent, version 18.0.0, adopting second order for all spatial discretization's and the SIMPLE scheme for pressure-velocity coupling. The convergence criterion for all the computed properties were established for residuals lower than $10^{-5}$.

\section{RESULTS AND DISCUSSION}

The GG model uses a constant absorption coefficient, this problem was solved for $\kappa=0.1 \mathrm{~m}^{-1}$. It is known that the realistic approach for absorption coefficient considers the molar concentrations of species and local temperature. However this simple model lead to reasonable results. Figure 2 shows the temperature in axial positions. Comparing with the experimental results it has a consistent behavior between the curves. However, changes are expected on temperature profile, but the temperature on center line did not show significant difference due to TRI influence. It is possible that the center line is not a good representative of the changes, but it was chosen due the locations of experimental data.

The profiles for temperature, Fig. 3 and Fig. 4, the rms temperature fluctuation, Fig. 5, are shown below. Reminding that the problem is axisymmetric. It can be seen that the temperatures profiles are similar. The rms value of the temperature fluctuation can reach the maximum value of approximately 
$300 \mathrm{~K}$, lower than the average flame temperature. This result was corroborated by Centeno, et al., (2016).

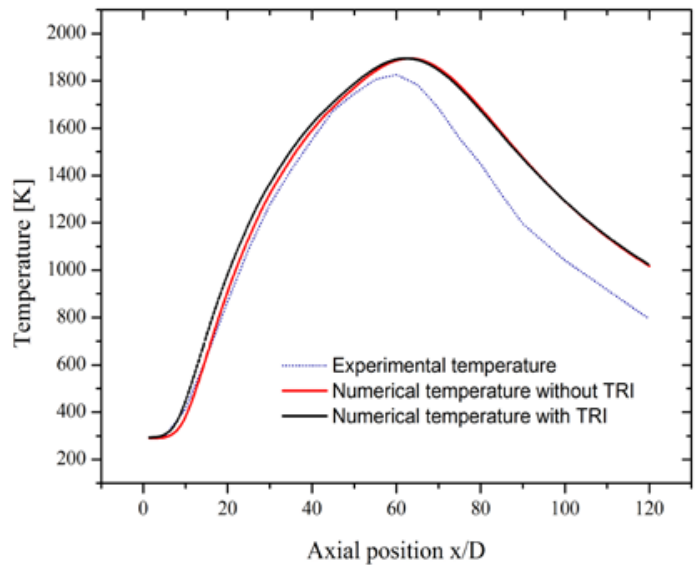

Figure 2. Comparison between numerical and experimental results.

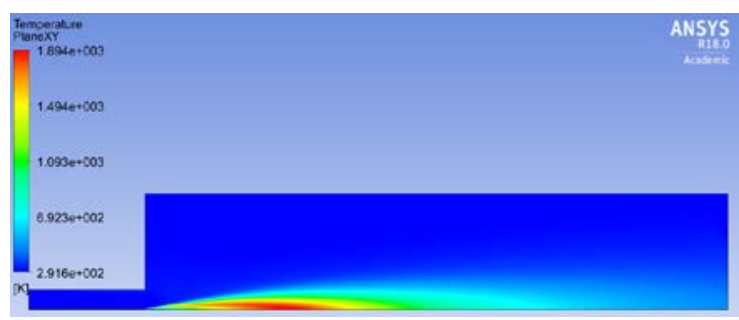

Figure 3. Temperature profile of flame DLR-A without TRI.

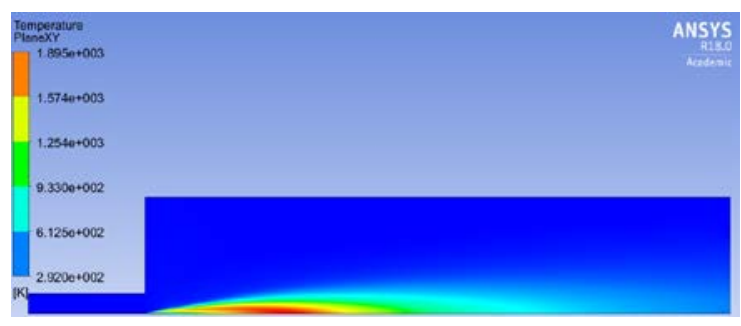

Figure 4. Temperature profile of flame DLR-A with TRI.

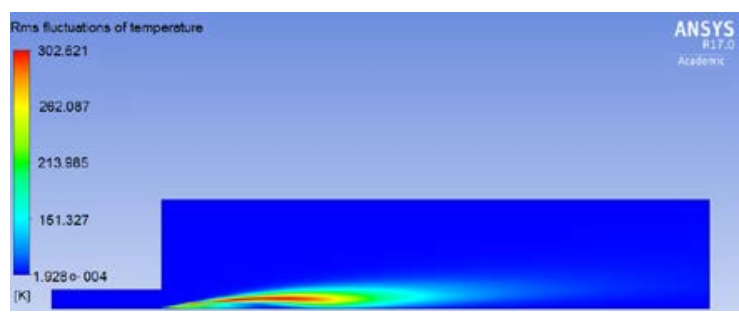

Figure 5. Rms fluctuations of temperature.

The radiative heat flux on the wall with coupling of TRI, Fig. 6, evidences the importance of considering the influence of TRI. This is a result without experimental data for comparison. The results are according with reviewers where the impact of TRI is $15 \%$ until $45 \%$ on radiative heat flux. The result obtained shown as average difference of 37\% between the solutions.

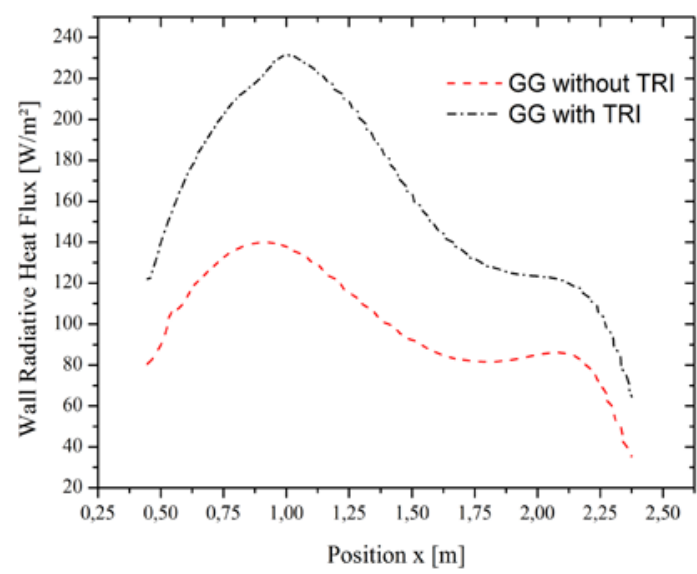

Figure 6. Wall radiative heat flux.

The species compared in this work are $\mathrm{CH}_{4}$, $\mathrm{CO}_{2}$ and $\mathrm{H}_{2} \mathrm{O}$, for study of this simulation. The comparison between the experimental and numerical data are shown on Fig.7, Fig.8 and Fig.9 with and without TRI.

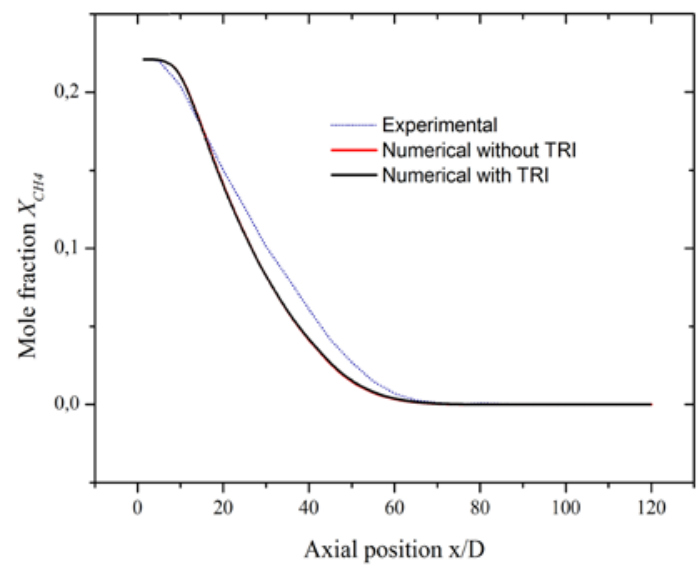

Figure 7. Comparison between $\mathrm{CH}_{4}$ evaluation.

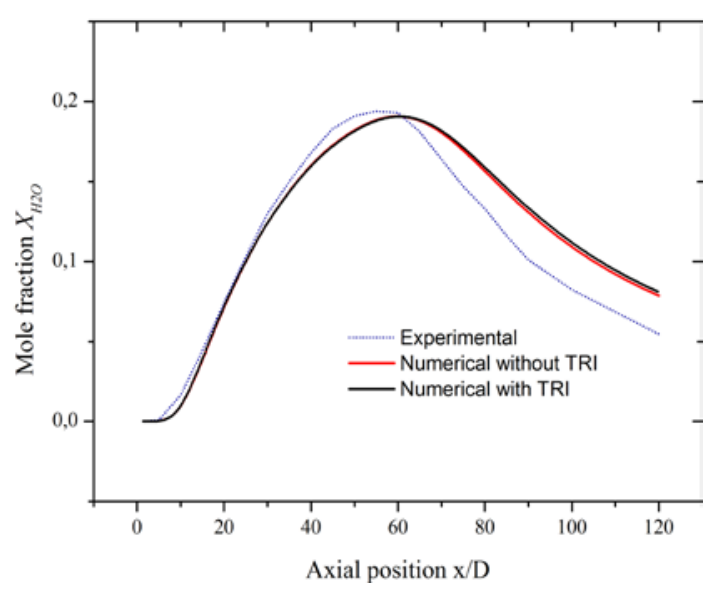

Figure 8. Comparison between $\mathrm{H}_{2} \mathrm{O}$ evaluation. 


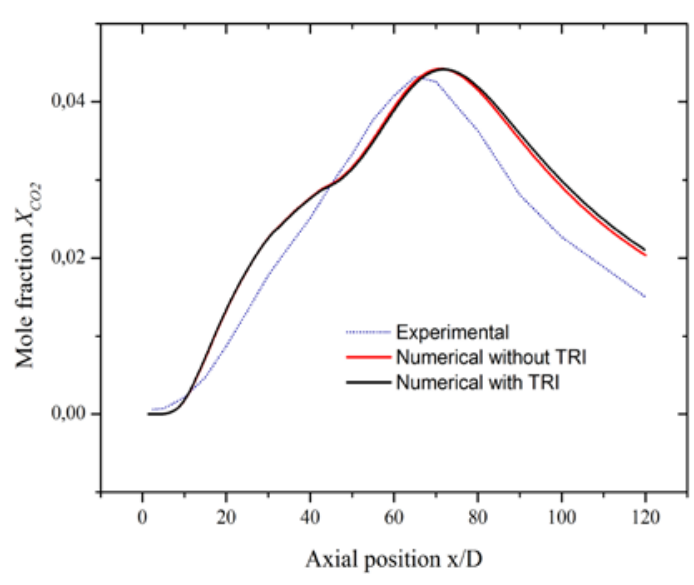

Figure 9. Comparison between $\mathrm{CO}_{2}$ evaluation.

The numerical results with and without TRI are close for species but the influence of TRI can be verified on radiative heat flux on the wall. Centeno et al. (2016) also reported a low impact of TRI on the prediction of chemical species, and the authors pointed to the global kinetic mechanism employed there. In the present investigation, the influence of TRI on the predictions of chemical species was low, but the kinetic mechanism is detailed (GRI-Mech 3.0 ), so it is a good indication that the influence of TRI on chemical species is negligible, independently from the combustion model considered.

\section{CONCLUSIONS}

This study applied the gray gas (GG) model for radiative heat flux, the SLDF and PDF table for chemical kinetic, $k-\varepsilon$ standard for turbulence, and Snegirev approach for TRI were applied o numerically solve the flame DLR-A, a non-premixed turbulent methane flame with air coflow. The objective was to analyze the influence of TRI on the main thermal parameters (temperature, chemical species and radiative heat fluxes). The obtained results showed a tendency of agreement with the experimental results available. For the employed models, chemical species and temperature were minor influenced by TRI, while its influence on radiative heat flux was important. The next study will consider the employment of WSGG model, for reducing the amount of simplifications regarding the absorption coefficient, which could bring the results closer to experimental data and additional insights about the TRI effects on the thermal behavior of this flame.

\section{ACKNOWLEDGEMENTS}

This work was supported by CNPQ research grants 309961/2013-0 and 476490/2013-8.

\section{REFERENCES}

Ansys, Fluent 14.0, 2011a, Theory Guide, Ansys Inc.

Ansys, Fluent 14.0, 2011b, UDF Manual, Ansys Inc.

Cassol, F., Brittes, R., Centeno, F., Silva, C. V., and França, F. H. R., 2015, Evaluation of the Grat Gas Model to Compute Radiative Transfer in NonIsothermal, Non-Homogeneous Participating Medium Containing $\mathrm{CO} 2, \mathrm{H} 2 \mathrm{O}$ and Soot, Journal of the Brazilian Society of Mechanical Sciences and Engineering, Vol. 37, pp.163-172.

Centeno, F. R., 2014, Modelagem da Radiação Térmica em Chamas Turbulentas da Combustão de Metano em Ar, Doctoral Thesis, UFRGS, Porto Alegre, RS. (in Portuguese)

Centeno, F. R., Brittes, R., and França F. H. R, 2016, Application of the WSGG Model for the Calculation of Gas-Soot Radiation in a Turbulent Non-Premixed Methane-Air Flame Inside a Cylindrical Combustion Chamber, International Journal of Heat and Mass Transfer, Vol. 93, pp. 7420-753.

Coelho, P. J., 2007, Numerical Simulation of the Interaction between Turbulence and Radiation in Reactive Flows, Progress in Energy and Combustion Science, Vol. 33, pp. 311-383.

Deon, D. L., 2016, Estudo Numérico de Chamas Turbulentas não Pré-Misturadas Através de Modelos Baseados no Conceito de Flamelets, Master Thesis, UFRGS, Porto Alegre. (in Portuguese)

Emami, M. D., and Fard, A. E., 2012, Laminar Flamelet Modeling of a Turbulent $\mathrm{CH} 4 / \mathrm{H} 2 / \mathrm{N} 2$ Jet Diffusion Flame Using Artificial Neural Networks, Applied Mathematical Modelling, Vol. 36, pp. 20822093.

Gupta, A., Haworth, D. C., and Modest, M. F., 2013, Turbulence-Radiation Interactions in LargeEddy Simulations of Luminous and Nonluminous Nonpremixed Flames, Proceedings of the Combustion Institute, Vol. 34, pp.1281-1288.

Li, G., and Modest, M. F., 2002a, Aplication of Composition PDF Methods in the Investigation of Turbulence-Radiation Interactions, Journal of Quantitative Spectroscopy and Radiative Transfer, Vol. 73, pp.461-472.

Li, G., and Modest, M. F., 2002b, Importance of Turbulence-Radiation Interactions in Turbulent Reacting Flows, in: ASME International Mechanical Engineering Congress and Exhibition, Louisiana, USA.

Meier, W., Barlow, R. S., Chen Y. -L., and Chen, J. -Y., 2000, Raman/Rayleigh/LIF Measurements in a Turbulent CH4/H2/N2 Jet Diffusion Flame: Experimental Techniques and Turbulence-Chemistry Interaction, Combustion and Flame, Vol. 123, pp. 326-343.

Peters, N., 1984, Laminar Diffusion Flamelet Models in Non-Premixed Turbulent Combustion, Progress in Energy and Combustion Science, Vol. 10, pp. 319-339. 
Schneider, Ch., Dreizler, A., Janicka, J., and Hassel, E. P., 2003, Flow Field Measurements of Stable and Locally Extinguishing HydrocarbonFuelled Jet Flames, Combustion and Flame, Vol. 135, pp. 185-190.

Snegirev, A. Y., 2004, Statistical Modeling of Thermal Radiation Transfer in Buoyant Turbulent Diffusion Flames, Combustion and Flame, Vol. 136, pp. 51-71.

TNF Workshop, 1998, Proceedings of the Third International Workshop on Measurement and Computation of Turbulent Nonpremixed Flames, Colorado, USA.

Ziani, L., Chaker, A., Chetehouna, K., Malek, A., and Mahmah, B., 2013, Numerical Simulations of Non-Premixed Turbulent Combustion of $\mathrm{CH} 4-\mathrm{H} 2$ Mixtures Using the PDF Approach, International Journal of Hydrogen Energy, Vol. 38, pp. 8597-8603. 